\title{
ÁREA DE ACCIÓN DEL CHIGÜIRO (Hydrochoerus isthmius) EN UN SISTEMA AGROPECUARIO EN CORDOBA, COLOMBIA
}

\section{HOME RANGE OF CAPYBARA (Hydrochoerus isthmius) LANDSCAPES IN LIVESTOCK IN CÓRDOBA, COLOMBIA}

\author{
CHACÓN, P. JULIO ${ }^{1 *}$ Biólogo., LINARES, A. JUAN ${ }^{1}$ MSc., CARRASCAL, V. \\ JUAN $^{2}$ MSc., BALLESTEROS, C. JESÚS ${ }^{1}$ MSc. \\ ${ }^{1}$ Facultad de Ciencias Básicas, Universidad de Córdoba. Grupo de \\ Investigación Biodiversidad Unicórdoba. Carrera 6 No. 76-103, Montería, \\ Córdoba, Colombia. ${ }^{2}$ Facultad de Medicina Veterinaria y Zootecnia, \\ Departamento de Ciencias Pecuarias, Universidad de Córdoba.
}

*Correspondencia: ichacon bio@hotmail.com

Recibido: 9-08-2013; Aceptado: 22-10-2013.

\section{Resumen}

El chigüiro es autóctono del continente americano; en Colombia se encuentran dos especies: Hydrochoerus hydrochaeris, con distribución en los Llanos Orientales de Colombia y Amazonas colombiano (también distribuido en Argentina, Paraguay, Ecuador, Venezuela, Brasil, Perú, Bolivia, las Guyanas); e Hydrochoerus isthmius, que habita al norte de Colombia, Panamá y Venezuela, aislada por los Andes de Mérida y la Serranía del Perijá. Este estudio buscó conocer el área de acción de $H$. isthmius, en paisajes ganaderos de Córdoba, al noroccidente de Colombia. Durante el período de marzo y mayo de 2007, dentro de predios ganaderos del municipio de Valencia, departamento de Córdoba, Colombia, se realizaron trabajos de seguimiento a individuos de dos grupos de una población de chigüiros. Se utilizó la técnica de radioseguimiento usando cuatro animales previamente radio-marcados. Se encontró que el área de acción de $H$. isthmius en paisajes de ganadería extensiva de Valencia, presenta una mayor área de acción durante la época seca $(1,93 \pm 0,88$ ha), comparada con la época de lluvias $(0,47 \pm 0,32 \mathrm{ha})$ en contraste con áreas de acción descritos en otros trabajos de $H$. hydrochaeris con reportes de áreas de uso que varían desde $5,5 \pm 10,8$ ha hasta 44 (10-56) ha, $H$. isthmius presenta áreas de acción más pequeños $(1,20 \pm 1,0$ ha) .

Palabras claves: Chigüiro, comportamiento animal, ganadería, telemetría, área de acción.

\section{Abstract}

The capybara is indigenous to the Americas, in Colombia there are two species: Hydrochoerus hydrochaeris, with distribution in the eastern plains of Colombia and Colombian Amazon (distributed also in Argentina, Paraguay, Ecuador, Venezuela, Brazil, Peru, Bolivia, Guyanas and eastern plains of Colombia); and Hydrochoerus isthmius, who lives in northern Colombia, Panama and Venezuela, isolated by the Andes of Merida and Serrania of Perija. This study sought to know the habitat range of $H$. isthmius in livestock landscapes of 
Córdoba, northwestern Colombia. During the period between march and may 2007, in cattle farms in the municipality of Valencia, department of Córdoba, Colombia, was carried out follow-up work to two groups of individuals of population of capybaras. We used radio-tracking technique using four animals previously radio-labeled. We found that home range of $H$. isthmius landscapes of extensive grazing of Valencia, presents a greater area of action during the dry season $(1,93 \pm 0,88 \mathrm{ha})$ compared to the rainy season $(0,47 \pm 0,32 \mathrm{ha})$ in contrast to habitat ranges described in other studies for the $\mathrm{H}$. hydrochaeris with reports of areas of use ranging from $5,5 \pm 10,8$ ha to $44(10-56)$ ha, $H$. isthmius presents smaller habitat ranges $(1,20 \pm 1,0 \mathrm{ha})$.

Key words: Capybara, animal behavior, livestock, telemetry, home range.

\section{Introducción}

El chigüiro está referenciado en la mayoría de la bibliografía como monotípico con una única especie Hydrochoerus hydrochaeris y dos subespecies $H$. $h$. hydrochaeris y H. h. isthmius (ALDANA-DOMÍNGUEZ et al., 2007). Sin embargo, Hydrochoerus isthmius descrito por Goldman (1912) es reconocida como especie (MONES, 1991; WILSON y REEDER, 2005, ALDANADOMÍNGUEZ et al., 2007). El chigüiro es utilizado como fuente de proteína en las comunidades indígenas y campesinas (ALHO 1986, MONES Y OJASTI 1986, FELDHAMER et al., 1999). Su capacidad reproductiva, alta tasa de crecimiento, rusticidad, comportamiento social y calidad de su carne, lo hace un recurso promisorio (ESCOBAR y GONZÁLEZ, 1976; BALLESTEROS y JORGENSON, 2009).

Los diversos hábitat que utiliza el chigüiro tienen como elementos comunes cuerpos de agua y zonas de pastoreo, que pueden ser pastizales naturales, potreros con pastos mejorados, bosques de galería, vegetación arbustiva o plantas emergentes de los cuerpos de agua (ALHO et al., 1989; BAPTISTE y FRANCO, 2006; ARTEGA y JORGENSON, 2007, BALLESTEROS y JORGENSON, 2009). Se le puede encontrar en ciénagas, pantanos, manglares salobres, meandros, esteros, orilla de ríos y canales de drenaje, bosques de galería y áreas abiertas con vegetación herbácea (OJASTI, 1973; MACDONALD, 1981; FELDHAMER et al., 1999; BALLESTEROS y JORGENSON, 2009). Es una especie bien adaptada a áreas de humedales y terrenos inundables, lo que le confiere alta eficiencia y ventaja competitiva en la utilización de forraje disponible en estos ambientes marginales a la ganadería (ESCOBAR y GONZÁLEZ, 1976).

En Colombia, algunas poblaciones silvestres de chigüiros son aprovechadas para la caza comercial o de subsistencia, pero no están establecidos protocolos de seguimiento y evaluación del comportamiento demográfico de las poblaciones (CAMARGO, 2005), con pocos estudios sobre el conocimiento del uso del espacio por parte de estos animales. Se reportan los trabajos de 
PERÉA y RUÍZ (1977) quienes describen un área de acción anual promedio de 44 ha para el departamento del Meta y 34 ha para la región del Arauca, Colombia. CAMARGO (2005), en un estudio realizado en el Municipio de Paz de Ariporo, Casanare, reporta un área de acción durante la época de lluvias de 32,74 ha; mientras que, para la época seca en una zona contigua, se reporta un área de acción de 6,19 $\pm 0,14$ ha (PARDO, 2006). ARTEGA y JORGENSON (2007) utilizando las huellas como patrón del uso del hábitat, realizaron un estudio en la Amazonía colombiana en busca de conocer los hábitos de desplazamiento del chigüiro; mientras que FERRAZ et al. (2007), en Piracicaba, Brasil, usando análisis de presencia/ausencia, estudiaron la distribución espacial teniendo en cuenta las características del hábitat en agroecosistemas.

Para el Caribe colombiano, son pocos los estudios acerca de la biología de $H$. isthmius; se resaltan los trabajos realizados por RUíZ (1989), DÍAZ (2007), GONZÁLEZ y NARANJO (2007), PATERNITA y CONSTAIN (2007), VILLADA y AHUMADA (2007), BALLESTEROS y JORGENSON (2009), CARRASCAL et al. (2011) y MIRANDA et al. (2011). Estos estudios no han profundizado en aspectos relacionados con el movimiento de la especie, conocimiento necesario para el establecimiento de planes de conservación y manejo; especialmente en el departamento de Córdoba, donde el chigüiro se considera como una especie de especial interés para la conservación (BAPTISTE y FRANCO, 2006). Este estudio tiene como objetivo determinar el área de acción del chigüiro en un ecosistema agropecuario de Córdoba, y relacionar los movimientos de los animales con aspectos determinantes en la producción agrícola y ganadera.

\section{Materiales y Métodos}

Área de estudio: El estudio se llevó a cabo durante las épocas de lluvias y seca entre los meses de marzo y mayo de 2007, en terrenos de la Hacienda Camagüey $\left(8^{\circ} 20^{\prime} \mathrm{N}\right.$ y $76^{\circ} 6^{\prime} \mathrm{W}$ ) en el Corregimiento de Villanueva, al noreste de la cabecera municipal de Valencia, Departamento de Córdoba, Colombia. La cobertura vegetal original de esta área correspondía al bosque seco tropical (HOLDRIDGE, 1967), donde procesos de transformación del paisaje han dado paso a sistemas de ganadería extensiva y pequeñas áreas de cultivos transitorios. Las áreas de potreros con pastos naturales se caracterizan por tener muy pocos árboles, algunas plantas herbáceas y gramíneas, donde se resaltan Sida glomerata, Borreria densiflora y Agrostis alba; con represas de almacenamiento de aguas lluvias (jagüeyes). La oferta de alimento está representada principalmente por la especie Bothriochloa pertusa, especie introducida que se ha expandido ampliamente en la costa Caribe colombiana, principal oferta de alimento y de un aporte nutricional importante para los chigüiros (GONZÁLEZ y NARANJO, 2007). En el sitio de estudio se encuentran 
pequeñas áreas con rastrojo bajo entramados con bejucos espinosos, que son utilizados como refugio para los animales, y una pequeña área con plantación forestal de Acacia mangium en las márgenes de los cuerpos de agua.

Métodos de campo: El área de acción y movimientos de los chigüiros se determinó aplicando la técnica de radio-seguimiento. Se utilizaron cuatro animales pertenecientes a dos grupos de una población de chigüiros, capturados en un corral cebado con maralfalfa (Pennisetum violaceum) y posteriormente sedados con una mezcla de $3 \mathrm{ml}$ de Ketamina $+1 \mathrm{ml}$ de Xilacina, revirtiendo el efecto con $2 \mathrm{ml}$ de Yohimbina, esto para seguridad en la manipulación e integridad de los animales, posterior a la captura fueron marcados con collares trasmisores marca ATS $\AA$. Para facilitar la recolección de los datos en campo, a los individuos marcados con radio-receptores se les asignó los nombres de: Apolo al macho dominante del primer grupo y Ceres a una hembra sub-adulta; Emira y Luisa a dos hembras adultas del segundo grupo. Se utilizaron dos receptores ATS $\AA$ R2100, dos antenas tipo Yagi y dos brújulas, con el fin de tomar azimuts desde estaciones distintas simultáneamente. El registro de los datos de azimuts y localización, teniendo en cuenta la dirección de la señal emitida por el collar, se realizó diariamente desde las 08:00 hasta las 17:00 horas, en intervalos de dos horas para cada animal. El seguimiento de Apolo y Ceres se realizó durante la época seca (marzo), mientras que el seguimiento de Emira y Luisa se llevó a cabo en la época de lluvias (mayo).

Análisis de los datos: La ubicación de cada animal se estimó por medio del programa LOCATE III (NAMS, 2006), que utiliza el estimador de Lenth para obtener las coordenadas estimadas de la posición del animal a partir de los azimuts tomados en campo y una elipse de error con un 95\% de confianza. Estas coordenadas se importaron a los programas ArcGis 9.2 y Ranges VI (KENWARD et al., 2003) y se calculó el área de acción usando el método de mínimo polígono convexo (PMC); y con la media armónica $(H c)$, se generaron los centros de actividad, un método que indica el centro real de actividad en el área de acción de los animales, movimientos desde áreas de menor frecuencia a áreas de mayor actividad o permanencia (DIXON y CHAPMAN 1980). De esta manera se calcularon áreas de acción con el 100\% y 95\% de los datos, así como un área núcleo con el $50 \%$ de las localizaciones, donde se observó una mayor frecuencia de uso por los animales.

El ámbito de hogar o área de acción promedio de los cuatro chigüiros radiomarcados es un promedio geométrico y se generó con el programa Microsoft ${ }^{\circledR}$ Office Excel 2007. Dado que el área tiende a tener distribución logarítmica, un promedio geométrico es más preciso que el calculado sin transformar las áreas individuales. Además, se generó un promedio aritmético con el fin de comparar con los datos obtenidos en trabajos realizados para otros hábitat con la especie 
H. hydrochaeris (PEREA y RUIZ, 1977; MCDONALD, 1981; HERRERA y MCDONALD, 1989; CAMARGO, 2005; PARDO, 2006; FERRAZ et al., 2007; CAMPOS, 2009; CORRIALE et al., 2013). Por otra parte, para evaluar la precisión del método utilizado y determinar si los errores del tamaño del área de la elipse de las localizaciones estaban asociados a los animales, o a la hora en que fueron tomados los datos, se compararon las áreas de las elipses con un test de Kruskal-Wallis utilizando el programa estadístico $R \circledast \quad(R$ DEVELOPMENT CORE TEAM, 2009) versión 2.10.1.

\section{Resultados}

Se registraron 142 localizaciones exitosas para los chigüiros radio-marcados con collar. Se obtuvo un distinto número de localizaciones por animal, ya que en algunos momentos no se recibía señal y fue imposible estimar los azimuts; además de la diferencia en el esfuerzo de muestreo entre los animales muestreados durante la época seca con respecto a los muestreados durante la época de lluvias. De este modo, de los animales Apolo y Ceres se registraron 53 localizaciones para cada uno; mientras que, para los animales Emira y Luisa se registraron 19 y 17 localizaciones, respectivamente.

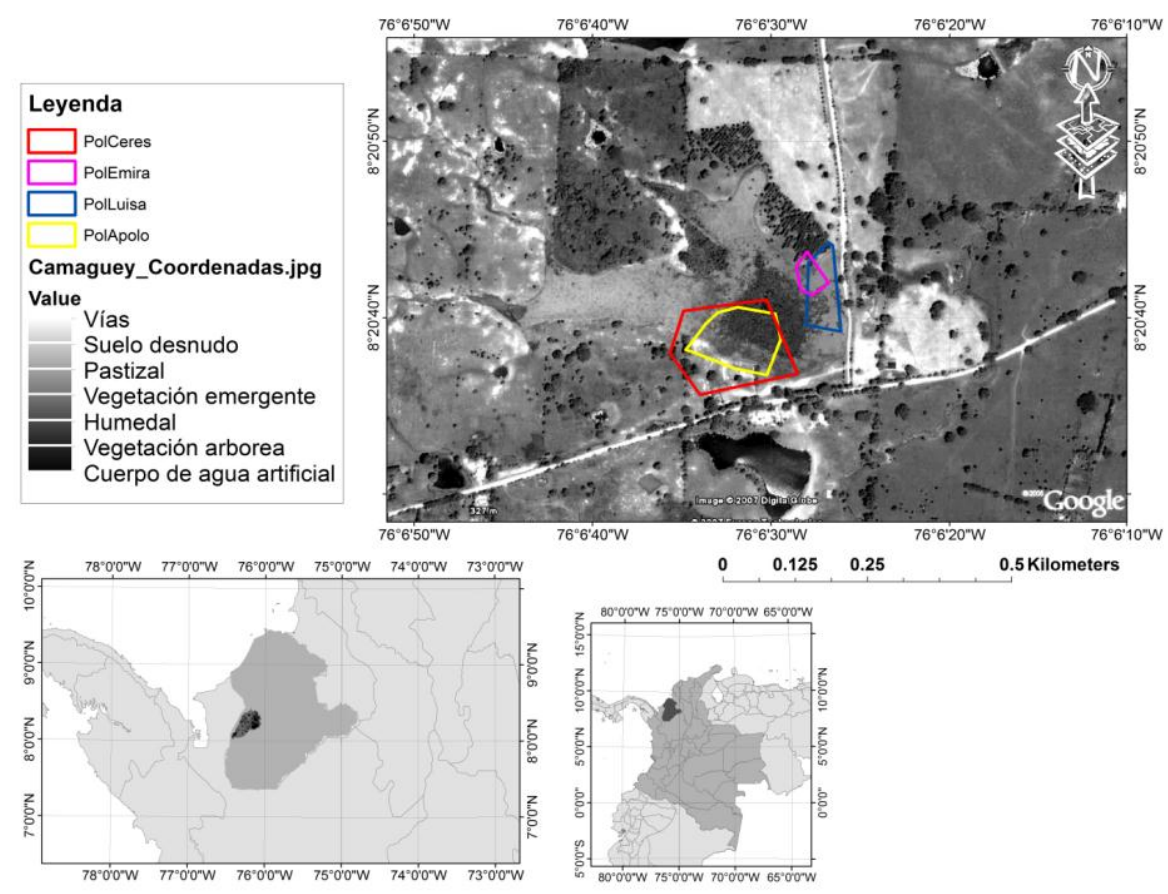

Figura 1. Área de acción del chigüiro (Hydrochoerus isthmius) en áreas de ganadería extensiva y sistemas de cultivos transitorios, en la Hacienda Camagüey, municipio de Valencia, Córdoba. Pol = mínimo polígono convexo (PMC).

El área de acción con promedio geométrico se estimó en $0,87 \pm 1,07$ ha, mientras que el promedio aritmético estuvo en $1,20 \pm 1,0$ ha (Fig. 1). Se encontraron valores estimados de las áreas de acción para cada animal, 
utilizando el $100 \%, 95 \%$ y $50 \%$ de las localizaciones, donde los animales con mayores áreas de acción fueron Apolo (1,31 ha) y Ceres (2,55 ha).

Para los períodos de lluvias y sequía se obtuvieron valores diferentes del área de acción de los animales. En la época de lluvias el área de uso de los chigüiros estuvo en un promedio de $0,47 \pm 0,32 \mathrm{ha}$; mientras que para la época seca se encontraron áreas de acción con promedio aritmético de 1,93 $\pm 0,88$ ha. Asimismo, se encontraron diferencias en los centros de actividad o área núcleo para los cuatro animales (Tabla 1), así: Apolo (0,36 ha), Ceres (0,25 ha), Emira $(0,03 \mathrm{ha})$ y Luisa $(0,18 \mathrm{ha})$. Al realizar el test de Kruskal-Wallis para comparar las áreas de las elipses de error, se encontró que el tamaño de estas áreas se encuentra influenciado por los animales $(p=0,00)$, mas no por las horas de realización del muestreo $(p=0,986)$, presentándose mayores áreas para el grupo de Apolo y Ceres (Fig. 2).

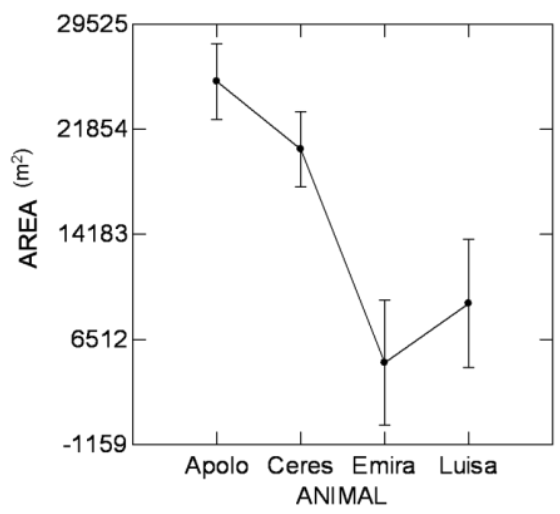

Figura 2. Promedios de las áreas de elipse de error asociadas a las localizaciones de cada chigüiro radio-marcado, en las épocas de lluvias y sequía, en un sistema de ganadería del Municipio de Valencia, Córdoba.

Tabla 1. Área de acción de los chigüiros (Hydrochoerus isthmius) en las épocas de lluvias y sequía, en un sistema de ganaderia en el Corregimiento de Villanueva, Municipio de Valencia, Córdoba.

\begin{tabular}{llcccc}
\hline Época & Animales & $\begin{array}{c}\text { No. de } \\
\text { localizaciones }\end{array}$ & $\begin{array}{c}\text { Localización } \\
\mathbf{1 0 0 \%}\end{array}$ & $\begin{array}{c}\text { Localización } \\
\mathbf{9 5 \%}\end{array}$ & $\begin{array}{c}\text { Área núcleo } \\
\mathbf{5 0 \%}\end{array}$ \\
\hline Seca & Apolo & 53 & 1,31 & 1,01 & 0,36 \\
& Ceres & 53 & 2,55 & 1,57 & 0,25 \\
Lluvia & Emira & 19 & 0,25 & 0,23 & 0,03 \\
& Luisa & 17 & 0,70 & 0,68 & 0,18 \\
\hline
\end{tabular}

En la tabla 2 se puede observar las diferencias en cuanto al área de acción de los individuos de $H$. hydrochaeris y los datos encontrados en el presente estudio. 
Tabla 2. Áreas de acción promedio reportados en la literatura para el chigüiro

\begin{tabular}{|c|c|c|c|c|c|c|}
\hline Localidad & $\begin{array}{l}\text { Época / } \\
\text { Estación }\end{array}$ & $\begin{array}{c}\text { Área de Acción } \\
\text { Promedio ha ( } \pm \\
\text { D.E) }\end{array}$ & $\begin{array}{c}\text { No de } \\
\text { animales }\end{array}$ & Técnica & Estimador & Fuente \\
\hline $\begin{array}{l}\text { Córdoba } \\
\text { (Colombia) }\end{array}$ & $\mathrm{S}-\mathrm{LI}$ & $1,20 \pm 1.0$ & 4 & $\mathrm{~T}$ & PMC & Este trabajo \\
\hline & $\mathrm{S}-\mathrm{LI}$ & $44(10-56)$ & 11 & $\mathrm{~T}$ & Área mínima & $\begin{array}{c}\text { PEREA y RUIZ } \\
(1977)\end{array}$ \\
\hline $\begin{array}{l}\text { Arauca } \\
\text { (Colombia) }\end{array}$ & $S-L I$ & $34(10-56)$ & 11 & $\mathrm{~T}$ & Área mínima & \\
\hline $\begin{array}{l}\text { Casanare } \\
\text { (Colombia) }\end{array}$ & LI & $\begin{array}{c}32,74(3,77- \\
54,48)\end{array}$ & 3 & $\mathrm{~T}$ & PMC & $\begin{array}{c}\text { CAMARGO } \\
(2005)\end{array}$ \\
\hline $\begin{array}{l}\text { Casanare } \\
\text { (Colombia) }\end{array}$ & S & 6,19 (0,14 SE) & 4 & $\mathrm{~T}$ & PMC & PARDO (2006) \\
\hline Venezuela & S & $5,5 \pm 10,8$ & N.E & OD & $N . E^{* *}$ & $\begin{array}{l}\text { McDONALD } \\
(1981)\end{array}$ \\
\hline Venezuela & $\mathrm{S}-\mathrm{LI}$ & $10,4 \pm 3,7$ & N.E & OD & $\begin{array}{l}\text { Polígonos } \\
\text { irregulares }\end{array}$ & $\begin{array}{c}\text { HERRERA y } \\
\text { McDONALD } \\
\text { (1989) }\end{array}$ \\
\hline Paraguay & $S-L I$ & $183 \pm 54$ & 6 & $\mathrm{~T}$ & Kernel 95\% & CAMPOS (2009) \\
\hline Argentina & $P$ & $8,05 \pm 4,25$ & N.E & OD & PMC & $\begin{array}{l}\text { CORRIALE et al. } \\
(2013)\end{array}$ \\
\hline Argentina & V & $6,95 \pm 3,48$ & N.E & OD & PMC & $\begin{array}{l}\text { CORRIALE et al. } \\
(2013)\end{array}$ \\
\hline Argentina & $\mathrm{O}$ & $11.64 \pm 4.2$ & N.E & OD & PMC & $\begin{array}{l}\text { CORRIALE et al. } \\
(2013)\end{array}$ \\
\hline Argentina & 1 & $13 \pm 5,97$ & N.E & OD & PMC & $\begin{array}{c}\text { CORRIALE et al. } \\
(2013)\end{array}$ \\
\hline
\end{tabular}

\section{Discusión}

El promedio aritmético del área de acción estimada presentó diferencias entre los animales estudiados. Sin embargo, todos los animales fueron observados alrededor del cuerpo de agua, realizando actividades tales como forrajeo y descanso en tierra. El área de acción de todo el grupo, estuvo influenciada por el humedal que les brindaba oferta de alimento y refugio contra los predadores.

Los dos grupos de animales presentan diferencias en cuanto a la distribución de sus manadas en el territorio disponible. En el caso de Emira y Luisa, se ubican bajo la sombra de plantaciones de Acacia mangium, y presentan cercanía con otro grupo en una distancia de unos $65 \mathrm{~m}$ y a una carretera que delimitaba su territorio durante el día. Por su parte, los animales Apolo y Ceres se encontraban ubicados en cercados con algunos árboles de matarratón (Gliricidia sepium), y presentan una distancia de unos $140 \mathrm{~m}$ con otro grupo; estas condiciones afectan el área de acción de cada una de las manadas de chigüiros, debido probablemente a la competencia entre machos dominantes por territorio y por fitness reproductivo en el caso de las hembras (HERRERA y MACDONALD, 1989). 
Es probable que la época de realización del muestreo, así como la cantidad de registros de localizaciones hayan influido en el resultado; lo que concuerda con lo descrito sobre el comportamiento de los animales durante las dos épocas climáticas, pues el hecho de haber menor oferta alimenticia durante la época seca (MACDONALD, 1981), provoca que los chigüiros salgan a buscar alimento ocupando un mayor territorio y dedicándole más tiempo a esta activida. ALHO et al. (1989), en el pantanal de Brasil, indican que el área de acción de los chigüiros es mayor durante la época seca; mientras que los trabajos realizados por PEREA y RUÍZ (1977), MACDONALD (1981), HERRERA y MACDONALD (1989) y PARDO (2006), reportan que el área de acción es mayor durante la época de lluvias.

Las diferencias entre los hábitat del chigüiro en Colombia, permite suponer que las poblaciones de la Región Caribe $(H$. isthmius), han estado sometidas a migraciones locales más cortas que las sufridas en los Llanos orientales por la especie $H$. hydrochaeris influido por su menor tamaño corporal (DÍAZ, 2007). La especie $H$. hydrochaeris tiene sus hábitat en humedales (esteros y morichales) con vastas áreas de bacines inundables aislados por grandes extensiones, sin vasos hidráulicos comunicantes, en paisaje de parches de humedales fragmentados en una matriz de sabanas. En contraste, $H$. isthmius en la Costa Caribe colombiana, sus hábitat corresponden a humedales en bacines inundables del sistema hidráulico de río conformando un paisaje que originalmente correspondía a una matriz de bosque seco tropical con parches de humedales conectados (BALLESTEROS y JORGENSON, 2009).

El análisis de los centros de actividad o áreas núcleo (áreas con el $50 \%$ de las localizaciones) para los cuatro animales evaluados, indican que el macho Apolo a pesar de presentar un área de acción menor (1,31 ha) con respecto a Ceres ( $2,55 \mathrm{ha})$, tiene un área núcleo mayor que el resto de animales. Esta situación se debe muy probablemente a que Apolo por su condición de macho dominante debe ocupar un área central mayor para cumplir con las funciones de territorialidad y el cuidado de las hembras del grupo (RAMíREZ, 2004).

Al contrastar las áreas de acción reportadas para la especie $H$. hydrochaeris (PEREA y RUIZ, 1977; MCDONALD, 1981; HERRERA y MCDONALD, 1989; CAMARGO, 2005; PARDO, 2006; FERRAZ et al., 2007, CAMPOS, 2009; CORRIALE et al., 2013), con los datos encontrados en este estudio para $H$. isthmius, se presentan claras diferencias, debidas probablemente a que el uso del espacio por los animales es el resultado de la combinación de factores internos (fisiológicos y morfológicos) del animal y factores externos del medio ambiente (BURT, 1943; GENTILE et al., 1997). De este modo, el área de acción está relacionada con las necesidades energéticas de las especies (LINDSTEDT et al., 1986), donde a mayor tamaño corporal más requerimientos energéticos, por lo cual, mayor área de acción (MCNAB, 1963; SWIHART et al., 
1988). Así, al ser $H$. hydrochaeris de mayor tamaño que $H$. isthmius (OJASTI, 1973; MONES y OJASTI, 1986; DÍAZ, 2007), esta requerirá más energía, en términos absolutos, para mantener su metabolismo (KOTLER et al., 1994), lo que condiciona una mayor área de acción.

El análisis de Kruskal-Wallis permite determinar que las áreas de los elipses de error se encuentran influenciadas por las condiciones ambientales, mas no por la horas de muestreo, resaltando que los grupos presentaron características particulares según su ubicación en el terreno, condiciones que pudieron influenciar probablemente las diferencias de las áreas de error; pues el grupo de Apolo y Ceres se encontraba más expuesto a fuentes de interferencia (tránsito de vehículos, antenas de televisión y radio, cercados, ondulaciones del terreno, entre otras); mientras que las estaciones del muestreo para el grupo de Emira y Luisa, no presentaban dicha condición. Así lo anotan SAMUEL y FULLER (1994), donde indican que los errores fundamentales del método telemétrico son las fuentes de interferencia y el tipo de antenas utilizadas (transmisora y receptora).

Agradecimientos: Al sistema agropecuario Camagüey en el municipio de Valencia por permitir la realización de esta investigación.

\section{Referencias}

ALDANA-DOMÍNGUEZ, J.; VIEIRA-MUÑOZ, M.I.; ÁNGEL-ESCOBAR, D.C. (eds.). 2007. Estudios sobre la ecología del chigüiro (Hydrochoerus hydrochaeris), enfocados a su manejo y uso sostenible en Colombia. Instituto Alexander von Humboldt. Bogotá D.C., Colombia.

ALHO, C. 1986. Criação e manejo de capybaras em pequenas propriedades rurais. EMBRAPA-DDT, Brasilia.

ALHO, C.J.R.; CAMPOS, Z.M.; GONÇALVES, H.C. 1989. Ecology, social behavior and management of the capybara in the Pantanal of Brazil. Pág. 163194. En: REDFORD, K.H.; EISENBERG, J.F. (eds.). Advances in Neotropical mammalogy. Sandhill Crane Press. Gainesville, E.U.

ARTEGA, M.; JORGENSON, J. 2007. Hábitos de desplazamiento y dieta del capibara (Hydrochoerus hydrochaeris) en la Amazonia Colombiana. Mastozoología Neotropical 14(1):11-17.

BALLESTEROS, J.; JORGENSON, J. 2009. Aspectos poblacionales del cacó (Hydrochoerus hydrochaeris isthmius) y amenazas para su conservación en el Nor-Occidente de Colombia. Mastozoología Neotropical 16(1):27-38.

BAPTISPE, M. P.; FRANCO, A. M. 2006. Especies focales para la conservación en el Departamento de Córdoba. Corporación Autónoma 
Regional de los Valles del Sinú y del San Jorge e Instituto de Recursos Biológicos Alexander Von Humboldt. Bogota D. C., Colombia.

BURT, W. H. 1943. Territoriality and home range concepts as applied to mammals. Journal of Mammalogy 24:346-352.

CAMARGO-SANABRIA, A. 2005. Área de acción y patrón de movimientos de tres manadas de Chigüiros (Hydrochaeris hydrochaeris) en el Hato Miramar como herramienta de manejo en el municipio de Paz de Ariporo, Casanare. Informe técnico. Universidad de los Llanos. Villavicencio, Colombia.

CAMPOS, J. M. 2009. Landscape ecology of the capybara (Hydrochoerus hydrochaeris) in the Chaco region of Paraguay. Ph.D. dissertation, Kansas State University, Manhattan.

CARRASCAL, J. C.; LINARES, J. C.; CHACÓN, J. 2011. Comportamiento del Hydrochoerus hydrochaeris isthmius en un sistema productivo del departamento de Córdoba, Colombia. Revista MVZ Córdoba 16(3):2754-2764.

CORRIALE, M.; MUSCHETTO, E.; HERRERA, E. 2013. Influence of group sizes and food resources in home-range sizes of capybaras from Argentina. Journal of Mammalogy 94(1):19-28.

DÍAZ, A. 2007. Caracterización Morfométrica del Chigüiro (Hydrochoerus hydrochaeris isthmius) en el Departamento de Córdoba, Colombia. Tesis de Pregrado. Universidad de Córdoba. Colombia.

DIXON, K.; CHAPMAN, J. 1980. Harmonic Mean Measure of Animal Activity Areas. Ecology 61(5):1040-1044.

ESCOBAR, A.; GONZÁLEZ-JIMÉNEZ, E. 1976. Estudio de la competencia alimentaria de los herbívoros mayores del llano inundable, con referencia especial al chigüire (Hydrochoerus hydrochaeris). Agronomía Tropical 26(3):213-227.

FELDHAMER, G.; L., DRICKAMER; S., VESSEY; J., MERRITT. 1999. Mammalogy: Adaptation, diversity and ecology. WCB/McGraw-Hill. New York, E.U.

FERRAZ, K. M. P. M.B.; FERRAZ, S. F.B.; MOREIRA, J. R.; COUTO, H. T. Z.; VERDADE, L. M. 2006. Capybara (Hydrochoerus hydrochaeris) distribution in agroecosystems: a cross-scale habitat analysis. Journal of Biogeography 34:223-230.

GENTILE, R.; D'ANDREA, P.; CERQUEIRA, R. 1997. Home Ranges of Philander frenata and Akodon cursor in a Brazilian Restinga (Coastal Shrubland). Mastozoología Neotropical 4(2):105-112.

GONZÁLEZ, O.; NARANJO, F. 2007. Caracterización poblacional, hábitos alimenticios y hábitat natural del chigüiro (Hydrochoerus hydrochaeris isthmius) 
en el Corregimiento de Villanueva, Municipio de Valencia, Departamento de Córdoba - Colombia. Tesis de Pregrado. Universidad de Córdoba. Colombia.

HERRERA, E.; MACDONALD, D.W. 1989. Resource utilization and territoriality in group-living Capybaras (Hydrochaeris Hydrochaeris). Journal of Animal Ecology 58:667-679.

HOLDRIDGE, L.R. 1967. Life Zone Ecology. Tropical Science Center.San José, Costa Rica. 206 pp.

KENWARD, R.E.; A.B, SOUTH; S.S, WALLS. 2003. Ranges6 v1.2: For the analysis of tracking and location data. Online manual. Anatrack Ltd. Wareham, UK.

KOTLER, B. P.; BROWN, J. S.; MITCHELL W. A. 1994. The role of predation in shaping the behavior, morphology and community organisation of desert rodents. Australian Journal of Zoology 42:449-466.

LINDSTEDT, S. L.; MILLER, B. J.; BUSKIRK, S. W. 1986. Home range, time and body size in mammals. Ecology 67:413-418.

MACDONALD D. 1981. Dwindling resources and the social behavior of capibaras (Hydrochoerus hydrochaeris) (Mammalia). Journal of Zoology 194(3):371-392.

McNAB, 1963. Bioenergetics and the Determination of Home Range Size. The American Naturalist 97:133-140.

MIRANDA, J.; CONTRERAS, V.; NEGRETE, Y.; MATTAR, S. 2011. Vigilancia de la infección por Rickettsia sp. en capibaras (Hydrochoerus hydrochaeris) un modelo potencial de alerta epidemiológica en zonas endémicas. Biomédica 31:216-221

MONES, A.; OJASTI, J. 1986. Hydrochoerus hydrochaeris. Mammalian Species 264:1-7.

MONES, A. 1991. Monografía de la familia Hydrochoeridae (Mammalia: Rodentia). Courier Forschungsinstitut Senckenberg 134:1-235.

NAMS, V. O. 2006. Locate III User's Guide. Pacer Computer Software, Tatamagouche, Nova Scotia, Canadá.

OJASTI, J. 1973. Estudio biológico del chigüire o capibara. Fondo Nacional de Investigaciones Agropecuarias, Caracas. Venezuela.

PARDO, L. 2006. Área de acción del Chigüiro (Hydrochoerus hydrochaeris), en el Municipio de Paz de Ariporo (Casanare), durante la época seca: Algunas consideraciones para su manejo. Tesis de grado. Universidad Nacional de Bogotá, Colombia. 
PEREA, J. T.; RUIZ, S. 1977. Organización social y hábitos territoriales del chigüiro. Tesis de Grado. Universidad Nacional de Bogotá, Colombia.

R DEVELOPMENT CORE TEAM. R: A language and environment for statistical computing [programa de ordenador]. Versión 2.10.1. R Foundation for Statistical Computing, Vienna, Austria. 2009.

RAMÍREZ-PERILLA, J. 2004. El chigüiro (Hydrochaeris hydrochaeris): Reflexiones acerca de las necesidades de conocimiento y tecnología para su gestión productiva sostenible. Departamento de Biología Universidad Nacional de Colombia. Pag. 62-82. En: Memorias encuentro latinoamericano sobre investigación y aprovechamiento sostenible de Hydrochoerus hydrochaeris. Yopal, Casanare, Colombia.

RUIZ, S. 1989. Estudio poblacional y del comportamiento del ponche (Hydrochaeris hydrochaeris isthmius). Tesis de grado. Sincelejo. Colombia: Universidad de Sucre.

SAMUEL, M. D.; FULLER, M. R. 1994. Wildlife radiotelemetry. Pag. 370-418. En: Bookhout, T. A. (ed.). Research and management techniques for wildlife and habitats. Fifth edition. The Wildlife Society. Bethesda, MD, E.U.

SWIHART, R. K.; SLADE, N. A.; BERGSTROM, B. J. 1988. Relating body size to the rate of home range use in mammals. Ecology 69, 393-399.

WILSON, D. E.; REEDER, D. M. (Eds). 2005. Mammal Species of the World: A Taxonomic and Geographic Reference. Third Edition. The Johns Hopkins University Press, Baltimore, USA. 